\title{
Editorial: The feasibility of the new sutureless excimer laser-assisted non-occlusive anastomosis clip in a rabbit model
}

\author{
Peter Vajkoczy ${ }^{1}$ (D) \\ Received: 13 October 2019 / Accepted: 14 October 2019 / Published online: 25 November 2019 \\ (C) Springer-Verlag GmbH Austria, part of Springer Nature 2019
}

Complex revascularization procedures sometimes have to go beyond the standard STA-MCA bypass procedure in order to deliver adequate amounts of blood flow, either for flow augmentation or flow replacement. Here, revascularization procedures with large caliber vessel grafts, such as the radial artery or saphenous vein, are applied. For those, the proximal anastomosis is typically located at the external carotid artery, the internal maxillary artery, or a prominent stump of the superficial temporal artery, and the distal anastomosis is located at the internal carotid artery of M1/M2 segments which will provide a good drain of the delivered blood. The typical use case for flow replacement with a large caliber graft is the reconstruction of the vascular tree in order to treat a complex aneurysm. The majority of complex aneurysms are giant, fusiform, or partially thrombosed/calcified, with the need for sacrificing the diseased vessel segment and revascularizing the dependent vascular territory with a bypass, as the only durable strategy for aneurysm exclusion.

The inherent risk of these intracranial anastomoses to proximal segments of major brain arteries is the need for temporary occlusion and concomitant temporary ischemia times. This is why the group around Prof Tulleken in Utrecht has pioneered techniques to circumvent temporary occlusion over the past decades, by creating an end-to-side anastomosis between large caliber grafts and major intracranial vessels. The first version that entered clinical application and trials was the ELANA (excimer laser-assisted non-occlusive anastomosis) procedure that does not require temporary occlusion of the recipient vessel, potentially allowing for a safer construction of high-flow EC-IC bypass grafts to large proximal arteries $(>2.6 \mathrm{~mm})$

This article is part of the Topical Collection on Vascular Neurosurgery -Other

Peter Vajkoczy

peter.vajkoczy@charite.de

1 Department of Neurosurgery, Charité-Universitätsmedizin Berlin, Charitéplatz 1, 10117 Berlin, Germany with resultant higher graft flows. Here, the saphenous vein is stitched on the recipient vessel using a platinum ring which flattens and stretches the vessel wall that has been selected as anastomotic site. Then, a laser catheter is inserted through the vein and vacuum suction is applied for $2 \mathrm{~min}$ in order to suck the vessel wall within the ring to the tip of the laser catheter. After a good contact between the laser catheter tip and vessel wall has been established, the flap within the ring is lasered out, without the need for temporary vessel occlusion. The ELANA technique has proven to work well for different surgical teams outside Utrecht $[1,2]$. A recent prospective multicenter IDE study on high-flow cerebral bypasses for anterior circulation artery aneurysms revealed 30-day morbidity and mortality rates of $19.4 \%$ and $5.6 \%$ in a difficult-to treat patient group [3]. These rates compare favorably with those rates of morbidity and mortality previously reported in the literature for conventional high flow bypass.

However, the ELANA procedure also carries some disadvantages. Among those is the surgical complexity and challenge of the procedure. The transsylvian working corridor is often deep and narrow while a minimum of eight microsutures are required to fix the device's platinum ring to the recipient vessel. Also, it not only about simply placing the sutures but also about placing the sutures in a way that guarantees even distribution of the tension forces on the vessel wall within the ring. Any flaws may lead to damage to surrounding tissues or unsuccessful retrieval of the vessel wall flap. Even in experienced hands, this technique affords a lot of training in the lab and still total suture time may last up to $90 \mathrm{~min}$.

This is why the Utrecht group has sought to develop a sutureless alternative for their laser-assisted technique of non-occlusive anastomosis between 2004 and 2010. The results on their first version were published in 2009 and the first clinical application was published in 2018 [4]. Here, the basic idea was to replace the sutured ring with a slide on which the graft is mechanically fixed and which is then pushed and anchored via two pins into the recipient vessel. This would fix and slightly stretch the recipient artery wall within the 
circumferential shape of the pins. Due to the lack of controlled sealing by sutures, the SELANA slide anastomosis had to be sealed with Tachosil or tissue glue. The 2018 paper demonstrated equivalency and even superiority to the conventional ELANA technique in terms of ease and patency in preclinical in vitro and in vivo models. Unfortunately, however, the translation of the SELANA slit technique into clinical practice failed after occurrence of major complications in the first patient related to injury to the recipient vessel wall and problems with sealing of the anastomotic site.

The present paper from de Boer and coworkers from the Utrecht group now takes the evolution of the SELANA technique to a next level [5]. The experiments presented in this experimental paper aimed at improving the reliability of the SELANA technique combining the slit/pin approach with a clip at the backside of the device as anchor and sealing mechanism. The new clip technique has been evaluated in a rabbit aorta model and has been compared with the ELANA technique. The results show that the SELANA clip technique is significantly easier and faster than ELANA while the flap rates were comparably successful in both groups with flap rates around $90 \%$. The qualitative analysis of the extent of perianastomotic hemorrhage suggested that the sealing of the anastomotic site has improved compared with the results with the slit technique, but still, external sealants may be necessary, in contrast to ELANA. The authors correctly conclude: The $\mathrm{SEcl}$ anastomosis is technically feasible in in vivo experiments. Mean application time, flap retrieval rate, hemostasis, and burst pressure are not inferior to the conventional ELANA anastomosis. Further long-term experiments should be performed to assess safety, patency, and re-endothelialization.

Overall, the clip technique described in this paper is another milestone in the evolution of non-occlusive bypass techniques. However, the past has shown that the translation of these techniques into clinical application is not straightforward. Vessel geometry and vessel wall quality of patients with cerebrovascular risk factors and disease are different from young and healthy rabbits. Also, the pitfalls of the surgical procedure are so manifold that it is almost impossible to foresee future challenges when the new technique will be applied in the human setting. The past has taught the authors and the community to stay critical and not to be overenthusiastic with these advancements. The honest and insightful 2018 report of the failed clinical application of the clip technique also raised the question of how the application of new (high-risk) techniques can be ethically justified in the light of having reliable conventional and ELANA anastomosis techniques in our armamentarium.

\section{References}

1. Langer DJ, Vajkoczy P (2005) ELANA: excimer laser-assisted nonocclusive anastomosis for extracranial-to-intracranial and intracranial-to-intracranial bypass: a review. Skull Base 15:191-205

2. Vajkoczy P, Korja M, Czabanka M, Schneider UC, Reinert M, Lehecka M, Schmiedek P, Hernesniemi J, Kivipelto L (2012) Experience in using the excimer laser-assisted nonocclusive anastomosis nonocclusive bypass technique for high-flow revascularization: Mannheim-Helsinki series of 64 patients. Neurosurgery 70:49-54

3. van der Zwan A, Langer D, Vajkoczy P, Welch B, Tymianski M, Kivipelto L, van Thoor S, Chakraborty S, O'Donnell D, Charbel F (2019) Final results of the prospective ELANA IDE study on high flow cerebral bypasses: anterior circulation aneurysms. Neurosurgery (in press)

4. van Doormaal TPC, de Boer B, Redegeld S, van Thoor S, Tulleken CAF, van der Zwan A (2018) Preclinical success but clinical failure of the sutureless excimer laser-assisted non-occlusive anastomosis (SELANA) slide. Acta Neurochir (Wien) 160:2159-2167

5. de Boer B, van Doormaal TPC, Stecher D, Redegeld S, Tulleken CAF (2019) Regli L, van der Zwan A. Feasibility of the new sutureless excimer laser assisted non-occlusive anastomosis clip in a rabbit model, Acta Neurochir (Wien) (in press)

Publisher's note Springer Nature remains neutral with regard to jurisdictional claims in published maps and institutional affiliations. 